\title{
Manfred Knapp
}

\section{Die Stimme Amerikas}

Auslandspropaganda der USA unter der Regierung John F. Kennedys.

Beiträge zur Sozialwissenschaftlichen Forschung Bd. 10, Opladen 1972

Westdeutscher Verlag, Bibliographie, Index, 199 S., DM 30

In den letzten Jahrzehnten haben sich folgenreiche Veränderungen in den Voraussetzungen, Instrumenten und Wirkungsmöglichkeiten, Inhalten und Zielen, Teilnehmern und Adressaten „,internationaler Kommunikation“ vollzogen. Sie ist kein Privileg staatlicher Funktionäre oder gesellschaftlicher Eliten mehr. Die staatlichen Hüllen sind jedenfalls in weiten Bereichen der Welt durchlässig geworden. Jeder Bürger kann aktiv oder passiv, bewußt oder unbewußt jederzeit Adressat, Träger oder Emittent von „internationaler" Kommunikation sein, der Student im Ausland, der Tourist, der Bürger, der einen ausländischen Film oder eine technische Ausstellung besucht. Die Veränderungen der Kommunikationstechnologien haben „instant communication“ eines jeden mit jedem überall in der Welt ermöglicht. Man sieht darin eine „Revolution“ des internationalen Verkehrs, die Entstehung einer „transnationalen Gesellschaft", und meint, Politik und ihr klassisches Instrument Diplomatie hätten abgedankt und würden durch „Volksdiplomatie“, „public diplomcay“ o. ä. ersetzt. - Nun, das alles sind Tendenzen, keine Zustände. Sicher ist, daß neue Formen, Techniken, Instrumente, Teilnehmer zwischenstaatlicher oder zwischengesellschaftlicher Kommunikation zusätzlich neben die alten Formen etc. getreten sind. Ein Ausschnitt aus diesem breit umschriebenen Problemfeld ist Propaganda, aber eben nur ein Ausschnitt. Propaganda wurde vereinfachend als der Versuch verstanden, im Ausland von der eigenen Nation/Staat, zumeist in Krisen- oder Kriegszeiten, ein möglichst günstiges Bild zu zeichnen, und zwar oft mit unlauteren (Wahrheits- oder Objektivitätsfrage) oder illegalen Mitteln. Über Propaganda in diesem Sinne gab es besonders in der Zeit zwischen den Weltkriegen schon Literatur, z. B. von John B. Whitton, oft unter (völker-) rechtlichen Aspekten. Es geht aber sachlich um mehr als um oft plumpe, gelegentlich intelligente Versuche der Selbstdarstellung, die in Einmischung in die Interna der Zielnation umschlagen. Wenn man Propaganda als Teil des eingangs angedeuteten erweiterten Kommunikationsfeldes zwischen Staaten und Gesellschaften auffaßt, wird sie zu einem höchst komplexen Problem. Soweit es sich um staatliche Aktivitäten der „Image-Pflege“ im Ausland handelt, ist das „Wahrheits“-Thema oft nicht das relevanteste. Vielmehr drücken sie auf differenzierte Weise das eigene ideologisch-gesellschaftliche Selbstverständnis und die entsprechenden Strukturen sowie die eigenen politischen und ideologischen Ziel- und Ordnungsvorstellungen aus, sie sind Bestandteil und Instrument der Gesamtheit dieser Politik, sie enthalten ein bestimmtes Vorverständnis über andere Gesellschaften; sie sollen Informationen über positive/negative Ergebnisse zurückmelden und sie in den eigenen Perzeptions- und politischen Entscheidungsprozeß rückkoppeln, wobei die Möglichkeit dazu wieder von den eigenen gesellschaftlichen usw. Prämissen und Strukturen abhängt. Beschäftigung mit Propaganda vermag also Erkenntnisse über ein neues „außenpolitisches“ Instrument per se, im übrigen aber über die Zielgesellschaft ebenso wie und vor allem über die emittierende Gesellschaft und die Gesamtheit ihrer (Außen-)Politik zu vermitteln. Sozialwissenschaftliche Studien mit dieser Zielsetzung gibt es bisher trotz der Wichtigkeit des Gegenstandes 
nicht viele. R. Doerries hat mit seiner Arbeit über deutsch-amerikanische Beziehungen 1912-1917 einen wichtigen historischen Beitrag dazu geleistet. Das Buch von M. Y. Cho über die „Volksdiplomatie“ enthält dazu erstaunlicherweise nichts, Krippendorffs "Amerikanische Strategie“ dagegen im Kapitel über das außenpolitische Instrumentarium einen Abschnitt über „Das demokratische Element: Propaganda." Als umfassende Monographie positiv zu erwähnen ist hier aber besonders D. Schröder: Die Volksdiplomatie (Den Haag 1972).

Das vorliegende Buch von Knapp (eine überarbeitete und s. Z. von E. O. Czempiel betreute Dissertation) hat sich zum Ziel gesetzt, diesen wichtiger werdenden, aber unzureichend analysierten Teilbereich amerikanischer Außenpolitik für die Kennedy-Ära systematisch zu untersuchen. Diese Phase ist relativ überschaubar und zeigt eine Verstärkung der fraglichen Aktivitäten.

Während einzelne amerikanische Staatsmänner, angefangen etwa mit B. Franklin, sich der Bedeutung der Image-Pflege im Auslande (Propaganda) durchaus bewußt waren (natürlich waren die gesellschaftlichen Wirkungsvoraussetzungen intern wie extern andere als heute), hat im amerikanischen Bewußtsein die auswärtige Politik seit 1776 eigentlich nur wirtschaftliche, militärische, allgemein-politische Dimensionen. Von einigen Episoden abgesehen (z. B. Wilsons Creel Committee), fingen die Amerikaner erst im 2. Weltkrieg an, systematisch und bewußt Propaganda zu betreiben. Hier setzt Knapp ein. Die beiden ersten Kapitel schildern von diesem Zeitpunkt an die historische Entwicklung bis zur Errichtung der USIA=U. S. Information Agency 1953 nebst den Außenstellen USIS=U. S. Information Service, Aufbau, Kompetenzen, Verhältnis zum State Department etc. Die nächsten beiden Kapitel haben die allgemeinen Aufgaben der USIA in ihrer neuen und im wesentlichen endgültigen Definition unter Kennedy und inhaltlich die einzelnen von der USIA entwickelten Programme jeweils entweder für bestimmte Adressaten (z. B. Westeuropa, 3. Welt - Afrika) oder für bestimmte Problembereiche (Kuba-Krisen, Mondfahrtprogramm) zum Gegenstand. Hier (Kennedys berühmte Anordnung von 1963, abgedr. S. 56) wird die neue Doppelrolle der USIA deutlich artikuliert: von der gewissermaßen klassischen „bloßen“ Nachrichtenagentur zu einer erweiterten, bisher teils umstrittenen, teils gar nicht deutlich artikulierten Rolle, nämlich "to help achieve U. S. foreign policy objectives by (a) influencing public attitudes in other nations, and (b) advising the President,... and the various departments... on the implications of foreign opinion for... U. S. policies...". Die USIA wird hier zwar deutlich als „Instrument“ (Knapp) im amerikanischen Außenpolitikprozeß verstanden und eingesetzt (was K. näher ausführt), zugleich gibt diese Formulierung aber das Selbstverständnis und die Intentionen führender Vertreter (bes. E. R. Murrow) der USIA und ihrer Befürworter, nämlich als mehr oder weniger selbständige Akteure im außenpolitischen Entscheidungsprozeß zu wirken (auch dazu gibt K. im einzelnen Auskunft), nur unvollständig wieder. Aus der Aktivierung und Dynamisierung der USIA-Arbeit ergeben sich gewisse Widersprüche und Konflikte zwischen lang- und kurzfristigen Zielen und entsprechenden Prioritäten, die in der Analyse deutlicher hätten verfolgt werden können. Denn einerseits geht es um die auswärtige Selbstdarstellung der USA im Sinne ihrer grundlegenden ideologischen Ziel- und Ordnungsvorstellungen und der Grundlagen ihres Gesellschafts- und Herrschaftssystems, zum anderen um die direkte, affirmative Darstellung und Unterstützung aktueller Politik und die Schwächung etwaiger kritischer Gegenpositionen. Gerade die im Hinblick hierauf vorgenommene Straffung, Kon- 
zentrierung und Schwerpunktbildung (62 f.) in der Ära Kennedy-Murrow führte innerhalb des Apparates zu Konflikten mit jenen, die sich an den längerfristigen Zielen und Mitteln (Kultur-Austausch usw.) orientieren.

Das 5. Kapitel untersucht dann die strukturellen und funktionellen Zusammenhänge sowie die Kommunikationsflüsse dieses „fourth arm of statecraft", dieser neuen psychologisch-ideologisch-politischen Dimension der Außenpolitik oder wie immer man sie bezeichnen will, und zwar im Verhältnis zwischen eigener amerikanischer Regierung und Gesellschaft wie zwischen ihnen und fremden Gesellschaften und Regierungen, ferner die Scheinalternative Propaganda-Information sowie das Verhältnis Propaganda-Kulturaustausch. Die letzten beiden Abschnitte schließlich sind (vom faktischen Informationsgehalt der ersten vier abgesehen) wohl die interessantesten. Hier setzt K. die amerikanische Propaganda in Beziehung einmal zum internationalen System (Kapitel 6); dessen Perzeption als einer ideologischen Polarisierung (Kalter Krieg, Kommunismus-Antikommunismus usw.) war für die bewußte Herausbildung und Verwendung des neuen Instruments entscheidend, weil trotz militärischer-wirtschaftlicher-politischer Erfolge in den USA große Unsicherheit bestehen blieb. K. weist mit Recht darauf hin (107), daß dieses Verhalten nicht zuletzt selbst den Kalten Krieg intensivierte. Man kann also von seinem sich selbst verstärkenden Regelkreis sprechen. K. deutet jedoch keine (theoretische oder praktische) Möglichkeit an, diesen Zirkel zu durchbrechen. Die amerikanische Diskussion (deren Argumentationsfront aufzubrechen $K$. nicht unternimmt) schwankt jedenfalls im Untersuchungszeitraum nur zwischen strikter Orientierung an Kaltem Krieg und Antikommunismus und einer etwas gemäßigteren aber immer noch intensiven ideologischen Konflikthaltung (Murrow) - das Schlachtfeld sind die „minds of men“, auf dem man sich dem ideologischen Gegner unterlegen fühlte und deshalb die eigenen Propagandaanstrengungen verstärkte. Nicht zuletzt infolge der Schwerfälligkeit des Apparates werden die zuletzt unter Kennedy einsetzenden vorsichtigen Wandlungen im Verhältnis zur Sowjetunion nur mit Verzögerung vom Propaganda-Instrument aufgenommen.

Der letzte Abschnitt endlich setzt die USIA konkret zum amerikanischen Gesellschafts- und Regierungssystem in Beziehung. Bestehen Entsprechungen? Insbesondere geht K. der Frage nach, wie weit Propaganda als außenpolitisches Instrument einer sich demokratisch verstehenden Gesellschaft „demokratische“ oder „undemokratische" Züge aufweist, und zwar sowohl im Hinblick auf die propagierende Gesellschaft wie auf die Wirkungen bei den Adressaten. Da der liberal-demokratische Demokratiebegriff existentiell auf „Offentlichkeit“ angewiesen ist, diese aber dem oft diskutierten "Strukturwandel“ (Habermas) unterliegt und zugleich Propaganda intern wie in ihren externen Wirkungen in diesem Zusammenhang zu sehen ist, liegt das Spannungsverhältnis zwischen ihr und Demokratie auf der Hand, ohne daß das Instrument per se "demokratisch“ oder "undemokratisch" wäre. Das hängt mehr von der Substanz der propagierten Politik und der jeweils konkreten Verwendung des Instruments ab. Das Problem einer „demokratischen Außenpolitik" (125 ff.), und zwar nicht nur im Sinne innenpolitischer demokratischer Legitimation und Kontrolle, sondern auch inhaltlich hinsichtlich ihrer Außenwirkung und Art der Exekution, schneidet K. leider nur an, ohne ihm theoretisch weiter nachzugeben, und zieht sich auf die Wiedergabe der inneramerikanischen Diskussion über das Problem zurück. - Ein Zusammenhang zwischen PropagandaInstrument und amerikanischer Gesellschaft besteht aber nicht nur im Demokratie- 
Kontext, sondern auch darin, daß Propaganda trotz ihrer Aufwertung unter Kennedy immer noch einen niedrigen Stellenwert im außenpolitischen Instrumentarium insgesamt hat. Die relativ bescheidenen Mittelbewilligungen für sie spiegeln die vergleichsweise höhere Einschätzung des militärischen oder wirtschaftlichen Armes der Außenpolitik in der amerikanischen Gesellschaft. Die Einstellung zur Propaganda als „salesmanship“ und die Verwendung ihrer Techniken, worauf $\mathrm{K}$. zu Recht hinweist, muß oft genug ihre externen Erfolgschancen beeinträchtigen und eben deshalb ihre Wertschätzung in einer Gesellschaft verringern, die meßbaren Erfolg erwartet. - Auch hier bleibt die Analyse etwas vordergründig. Dieser Sachverhalt hätte Überlegungen zum Mangel oder Verlust an Lernfähigkeit der amerikanischen Gesellschaft nahegelegt. Denn wenn auch die Steigerung der Propaganda-Aktivitäten aus einem subjektiven Gefühl der Bedrohung entstanden sein mag, reflektieren diese objektiv doch gerade die (jedenfalls damals noch vorhandene) Mächtigkeit der USA, denn nur der Mächtige kann es sich leisten, nicht $\mathrm{zu}$ lernen und sich nicht $\mathrm{zu}$ adaptieren, diese Last also anderen aufzuzwingen, aber auch das nur bis zu einem Punkt des Bruches oder Umschlages.

So sind insgesamt die eingangs angedeuteten Fragen zu einem so wichtigen und interessanten Gegenstand oft mehr angerissen als durchgeführt. Gerade die theoretische Reflexion bricht verschiedentlich dort $a b$, wo sie interessant zu werden beginnt. Diese Kritik erhebt sich für den, der mit der Materie einigermaßen vertraut ist. Als Einführung in sie und als innerhalb der selbstgesteckten Grenzen ausgezeichnet, materialreich und informativ gearbeitete Studie ist das Buch jedem Interessierten bestens zu empfehlen.

Knud Krakau

\section{Siegfried Magiera}

Die Vorwahlen (Primaries) in den Vereinigten Staaten

Demokratisierung von Wahlen und Parteien. Parlamente und Parteien Bd. 3, hrsg. vom Institut für Internationales Recht an der Universität Kiel, Frankfurt 1971, Athenäum Verlag, 174 S. Bibliographie, Index

\section{EBERHARD KÖLSCH}

\section{Vorwahlen}

Zur Kandidatenaufstellung in den USA. Berlin 1972, Duncker und Humblot, Schriften zum Offentlichen Recht, Bd. 187, 166 S. Bibliographie, kein Index

Diese beiden Arbeiten, fast gleichzeitig entstanden, fordern zum Vergleich heraus, da sie denselben Gegenstand behandeln: die amerikanischen Vorwahlen (Vw). Die Vw spielen übrigens für die Präsidentenwahlen, wodurch sie jedem Zeitungsleser auch außerhalb der USA geläufig sind, nur eine untergeordnete und indirekt wirkende Rolle. Obwohl beide Arbeiten von einem juristischen Verlag bzw. Institut in Reihen herausgegeben worden sind, die im allgemeinen juristische Arbeiten (meist Dissertationen) betreuen, stellt sich dieser Vergleich als nicht sehr produktiv heraus, weil es sich um sehr unterschiedliche Ansätze und deshalb praktisch inkommensurable Arbeiten handelt. Das Buch von Kölsch ist im wesentlichen das eines Politikwissenschaftlers, der sich auf moderne Wahlanalysen mittels quantifizierender, statistischer Methoden stützt und sie referierend, interpretierend, die Daten auch neu verarbeitend fortführt. Es ist in erster Linie analytisch, systema- 